\title{
Vaginal Leiomyoma in a Post Menopausal Woman: Unusual Case
}

\author{
B.J. Kalibushi ${ }^{1 *}$, G.J. Gilson ${ }^{2}$ and E. Habimana ${ }^{3}$ \\ ${ }^{1}$ Department of Obstetrics and Gynecology, Butare University Teaching \\ Hospital (BUTH), Rwanda \\ ${ }^{2}$ HRH Rwanda Staff, Maternal Fetal Medicine Specialist, BUTH, Rwanda \\ ${ }^{3}$ Obstetrician and Gynecologist, BUTH, University of Rwanda, Rwanda \\ *Corresponding author
}

\section{A B S T R A C T}

Vaginal leiomyoma is a rare tumor with a variable clinical presentation and a broad differential diagnosis that can be confounded with pelvic organ prolapse, as in this case. We present a case of vaginal leiomyoma without urinary symptoms but with mild vaginal

\section{Keywords}

Vaginal leiomyoma, Surgical enucleation.

Article Info

Accepted:

28 October 2017

Available Online:

10 December 2017 discomfort. A 59-year-oldG4P4 postmenopausal woman presented with a 5-year history of progressive sensation of prolapse and recurrent vaginal discharge, but without urinary tract symptoms. She was referred with a diagnosis of stage 3 uterine prolapse. Simple external genital examination revealed a smooth mobile $6.5 \times 4.5 \mathrm{~cm}$ vaginal mass arising from the anterior vaginal wall, inferior and lateral to the urethra. Digital examination revealed a solid, mobile, paraurethral, vaginal mass, without evidence of pelvic relaxation. Ultrasonography and selective hematology work up were normal. Under spinal anesthesia, the mass was enucleated through a vertical incision, the urethra being continuously protected by inserting a Foley catheter and retracting it laterally to avoid inadvertent urethral compromise. Histology confirmed a benign leiomyoma. The evaluation of an anterior wall vaginal mass must include a thorough pelvic examination and may require urethrocystoscopy or other investigations such as positive-pressure urethrography. Surgical enucleation via a vaginal approach is the treatment of choice. Histology should be sent for precise identification of the mass.

\section{Introduction}

Vaginal leiomyoma is a rare tumor with a variable clinical presentation and a broad differential diagnosis that can be confounded with pelvic organ prolapse, as in this case.

The vaginal leiomyoma is an uncommon type of fibroid with usually anterior vaginal wall location (1). (2)

In low resources setting, efficient pelvic examination will rule out the pelvic organ prolapse. We are reporting this case of vaginal leiomyoma which was misdiagnosed, for helping health provider to be away to this rare vaginal tumor as one of differential diagnosis of uterine prolapse and cystocele.

\section{Case presentation}

A 59-year-oldG4P4 postmenopausal woman presented with a 5-year history of progressive sensation of prolapse and recurrent vaginal 
discharge, but without urinary tract symptoms. She was referred with a diagnosis of stage 3 uterine prolapse from district hospital.

At the university hospital a simple external genital examination revealed a smooth mobile $6.5 \times 4.5 \mathrm{~cm}$ vaginal mass arising from the anterior vaginal wall, inferior and lateral to the urethra. Digital examination revealed a solid, mobile, paraurethral, vaginal mass, without evidence of pelvic relaxation.

Ultrasonography and selective hematology work up were normal. Under spinal anesthesia, the mass was enucleated through a vertical incision, the urethra being continuously protected by inserting a Foley catheter and retracting it laterally to avoid inadvertent urethral compromise.

She was discharged home on the second postoperative day without treatment. Histology confirmed a benign leiomyoma.

The evaluation of an anterior wall vaginal mass must include a thorough pelvic examination and may require urethrocystoscopy or other investigations such as positive-pressure urethrography. Surgical enucleation via a vaginal approach is the treatment of choice. Histology should be sent for precise identification of the mass.

\section{Results and Discussion}

About 330 cases have been reported since the first detected case back in 1733 by Denys de Leyden (3). They are varied clinical presentation, the most common being vaginal mass $(4,5)$

Vaginal leiomyomas usually develop in women between the age of 35 and 50(6), but in our case, it was seen at age 54 with progressive growth and it was been diagnosed 5 years later. That age rang was similar to observation indicted that the vaginal myomas have been reported in patients from puberty to 71years of age (7).

Average age of diagnosis tends to be in late 30 or early 40 which was contrary to our findings. The diagnosed age was at 59 years without urinary tract symptoms as described as common symptomatology in those cases (7). However, many patients only describe a bulging mass (7) as in our case report.

\section{Vaginal leiomyoma in a post menopausal woman}

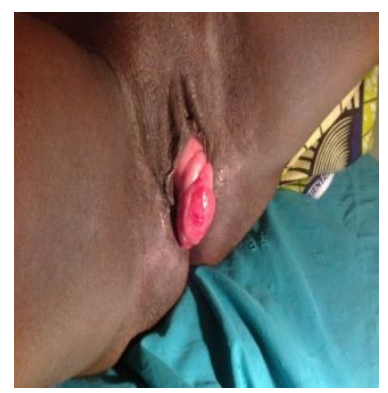

These lesions are usually estrogen dependent and can grow rapidly during pregnancy or regress after menopause (6) which explained the slowdown in growth of our case of vaginal leiomyoma. Before surgery, in low resources setting an appropriate pelvic examination for localization and exclusion of some pelvic organ prolapse and ultrasound may help to estimate the extent because we didn't have sophisticated equipment as MRI, urethrocystoscopy or other investigations such as positive-pressure urethrography. 
Histology is the final investigation for confirming the diagnosis. Enucleation through a vaginal approach was done successful without urethra injury as curative treatment.

\section{Conflict of Interest}

The authors declare they have no conflict of interest

\section{Acknowledgment}

The authors expressed their gratitude to the patient for consenting to reveal the case details and photographs for publication.

\section{References}

1. Smitha V Nidhanee et al., An unusual presentation of a vaginal leiomyoma in a postmenopausal hysterectomised women: a case report. Cases Journal 2009, 2: 6461. doi:10.1186/1757-1626$2-6461$
2. Kaufman RH, Gardner HL: Benign mesodermal tumors. Aust NZ Obstet Gynaecol 1996, 36: 494-495

3. Young SB, Rose PG, Reuter KL, vaginal fibromyomata: two cases with preoperative assessment, resection and reconstruction. Obstet Gynecol 1991 Nov; 78(5pt2): 972-974

4. Ruggieri AM, Brody JM, Curhan RP: Vaginal leiomyoma. A case report with imaging findings. J Reprod Med 1996, 41: 875-877.

5. Neil CP, Tiltman AJ: Leiomyoma Of vagina. S Afr Med J 1978, 54:816-817.

6. Costantini E., Cochetti G., Porena M. Vaginal para-urethral myxoid leiomyoma: case report and review of the literature. Int Urogynecol J Pelvic Floor Dysfunct. 2008; 19(8): 11831185.

7. Pulfus E, Newcomer J: Vaginal wall mass: Guest editorial. Obstetrical and gynaecological survey 1999, 54: 149150.

\section{How to cite this article:}

Kalibushi, B.J., G.J. Gilson and Habimana, E. 2017. Vaginal Leiomyoma in a Post-Menopausal Woman: Unusual Case. Int.J.Curr.Microbiol.App.Sci. 6(12): 4232-4234. doi: https://doi.org/10.20546/ijcmas.2017.612.487 